\title{
On the Micromechanisms of Shock-Induced Martensitic Transformation in Tantalum
}

L. L. Hsiung

December 15, 2005

2005 14th APS Topical Conference on Shock Compression of Condensed Matter Baltimore, MD, United States July 31, 2005 through August 5, 2005 
This document was prepared as an account of work sponsored by an agency of the United States Government. Neither the United States Government nor the University of California nor any of their employees, makes any warranty, express or implied, or assumes any legal liability or responsibility for the accuracy, completeness, or usefulness of any information, apparatus, product, or process disclosed, or represents that its use would not infringe privately owned rights. Reference herein to any specific commercial product, process, or service by trade name, trademark, manufacturer, or otherwise, does not necessarily constitute or imply its endorsement, recommendation, or favoring by the United States Government or the University of California. The views and opinions of authors expressed herein do not necessarily state or reflect those of the United States Government or the University of California, and shall not be used for advertising or product endorsement purposes. 


\title{
ON THE MICROMECHANISMS OF SHOCK-INDUCED MARTENSITIC TRANSFORMATION IN TANTALUM
}

\author{
Luke L. Hsiung \\ Lawrence Livermore National Laboratory \\ P. O. Box 808, L-352 \\ Livermore, CA 94551-9900, USA
}

\begin{abstract}
Shock-induced twinning and martensitic transformation in tantalum, which exhibits no solid-state phase transformation under hydrostatic pressures up to $100 \mathrm{GPa}$, have been further investigated. Since the volume fraction and size of twin and phase domains are small in scale, they are considered foming by heterogeneous nucleation that is catalyzed by high density lattice dislocations. A dynamic dislocaion mechanism is accordingly proposed based upon the observation of dense dislocation clustering within shock-recovered tantalum. The dense dislocation clustering can cause a significant increase of strain energy in local regions of $\beta$ (bcc) matrix, which renders mechanical instability and initiates the nucleation of twin and phase domains through the spontaneous reactions of dislocation dissociation within the dislocation clusters. That is, twin domains can be nucleated within the clusters through the homogeneous dissociation of $1 / 2<111>$ dislocations into $1 / 6<111>$ partial dislocations, and $\omega$ phase domains can be nucleated within the closters through the inhomogeneous dissociation of $1 / 2<111>$ dislocations into $1 / 12<111>, 1 / 3<111>$ and $1 / 12<111>$ partial dislocations.
\end{abstract}

Keywords: Shock compression, tantalum, dislocations, twinning, phase transitions: bcc crystals PACS:62.50.+P, 64.60.-i.

\section{INTRODUCTION}

It is well known that the combined effects of high pressure, high strain rate, and high plastic strain on tantalum can generate a broad range of microstructural features such as dislocation cells/walls, subboundaries, adiabatic shear bands, recrystallized grains, and deformation twins... etc. [1-6]. A recent TEM study of deformation substructure developed within shock-compressed tantalum has revealed that shock-induced phase transformation can take place in polycrystalline tantalum and tantalum-tungsten alloys shock-compressed at $50 \mathrm{GPa}$, but it does not take place in [011]-oriented single-crystal tantalum shockcompressed under the same conditions [7, 8]. Noted that tantalum exhibits no equilibrium solid-state phase transformation under hydrostatic pressures below 100 GPa $[9,10]$.

It has been proposed that the $\omega$-lattice can be obtained by collapsing one pair of (111) planes within the $\beta$-lattice and leaving the adjacent (111) planes unaltered [11]. The atomic displacements required are $\pm a_{0} \sqrt{3} / 12$, where $a_{o}$ is the lattice parameter of $\beta$-lattice. However, the collapse is not always complete, and it may result in a "rumpled" plane after collapsing. The crystal structure of ideal $\omega$ is hexagonal if the collapse is complete. Both ideal and rumpled hexagonal contain 3atoms/unit cell, the equivalent positions are: $0,0,0$; $2 / 3,1 / 3,1 / 2 ; 1 / 3,2 / 3,1 / 2$ for ideal hexagonal, and $0,0,0$; $2 / 3,1 / 3,1 / 2+\mathrm{z} ; 1 / 3,2 / 3,1 / 2-\mathrm{z}(0<\mathrm{z}<0.167)$ for non-ideal hexagonal or rumpled structure. It has been proposed previously [8] that similar to a shear mechanism for deformation twinning, i.e. the movements of a homogeneous 1/6[111] shear in consecutive (211) planes; omega phase can be formed by an inhomogeneous shear of $1 / 12[111], 1 / 3[111$ ], and $1 / 12[111$ ] in consecutive $\{211\}_{\beta}$ planes. The omega sequence caused by the threelayer inhomogeneous shear is equivalent to the atomic - -

shuffling of $\pm 1 / 12[111]$ in a pair of $\{211\}$ planes, which transforms the bcc lattice to the omega lattice based upon the collapse model. The shock-induced $\beta \rightarrow \omega$ transition in tantalum can therefore be regarded as a diffusionless martensitic transformation. 
The $\beta \rightarrow \omega$ transition occurs in shock-compressed polycrystalline tantalum at $50 \mathrm{GPa}$ but does not occur in single-crystal tantalum shock-compressed at $50 \mathrm{GPa}$ and static-compressed below $100 \mathrm{GPa}$ reveals that a unique reaction has occurred in polycrystalline tantalum under dynamic-pressure conditions. It is known that the phase stability is mainly governed by its thermodynamic free energy represented by the equation of state (EOS) for which the free energy of the phase that is the lowest will be stable. In the case of pressure-induced phase transformation under hydrostatic pressure conditions, a material can undergoe structure or phase change when the free energy of competing phase becomes smaller than that of the parent phase above a citical pressure. The equilibrium $\beta \rightarrow \omega$ phase transition occurs when the pressure increases above a critical pressure. However, unlike hydrostatic pressure, dynamic pressure can lead to a considerable increase in temperature; the higher the applied pressure, the higher the overheat temperature. This means that a higher pressure is required to achieve an equal volume or density of a compressed material in a shock experiment than in a hydrostatic experiment. Accordingly, the equilibrium transition pressure is anticipated to increase in material compressed under dynamic-pressure conditions than that compressed under hydrostatic-pressure conditions. Although no report on equilibrium transition pressure under hydrostatic pressure conditions is available for tantalum which exhibits no solid state phase transformation under hydrostatic pressures below 100 $\mathrm{GPa}[7,8]$, it is reasonable to postulate that the equilibrium transition pressure for tantalum under dynamic pressure conditions will be considerably higher than 100 GPa because of the temperature effect. The observation of $\beta \rightarrow \omega$ transition in shockcompressed tantalum and tantalum-tungsten alloys at 50 GPa therefore reveals that a nonequilibrium phase transformation has taken place at such a pressure below $100 \mathrm{GPa}$.

It is accordingly postulated that the EOS derived from hydrostaic experiment may not be applicable for materials compressed under dynamic pressure conditions because of the occurrence of shock-induced nonequilibrium phase transformation. That is, the EOS based upon macroscopic thermodynamics, which asserts that the system free energy $(\mathrm{G})$ is a function of volume $(\mathrm{V})$, pressure $(\mathrm{P})$, and temperature $(\mathrm{T})$, i.e., $\mathrm{G}$ $=\mathrm{F}(\mathrm{V}, \mathrm{P}, \mathrm{T})$ is insufficient to rationalize the system free energy under dynamic-pressure conditions. Additional state variables or factors are required to depict the free energy function governing the phase stability under dynamic pressure conditions. Since shear deformation has been found to play a crucial role in the formation of shock-induced $\omega$ phase in tantalum [8], it is accordingly suggested that high density and unique configuration of dislocation structure generated under dynamic pressures can also contribute considerably to phase stability of shock-compressed tantalum. The objective of this investigation is accordingly aimed at investigating the unique dislocation configurations developed within shockcompressed single-crystal and polycrystalline tantalum in order to depict the micromechanisms of shockinduced non-equilibrium phase transformation.

\section{EXPERIMENTAL PROCEDURE}

Ingot metallurgy (IM) tantalum in the form of plate stock was obtained from Cabot Corporation, Boyerstown, PA and was produced using a standard electron-beam melt process. The interstitial impurities in this test material are, in parts per million (ppm), oxygen: 23; nitrogen: 20; hydrogen: 1.3 ; carbon: $<2$. A single explosively driven shock-recovery experiment was performed in which cylindrical samples of $5 \mathrm{~mm}$ thick, $40 \mathrm{~mm}$ in diameter were used for the test and the loading axis is parallel to the cylindrical axis. The calculated peak shock pressure and duration for the shock-recovery test were $50 \mathrm{GPa}$ (a correction from previously reported $45 \mathrm{GPa}$ ) and $1.8 \mu \mathrm{s}$, respectively. Microstructures of shock-recovered [011]-oriented singlecrystal and polycrystalline samples were examined using a JEOL-200CX transmission electron microscope. TEM specimens were finally prepared by a standard twin jet electropolishing technique in a solution of 94 vol.\% methanol, 5 vol.\% sulfuric acid and 1 vol.\% hydrofluoric acid at $-25 \mathrm{~V}$ and $-20^{\circ} \mathrm{C}$.

\section{RESULTS AND DISCUSSION}

\section{Identification of shock-induced omega phase in polycrystalline tantalum}

Figure 1 shows the coexistence of (211)-type twin domains with fine $\omega$ plates observed within a shockrecovered polycrystalline sample. Here, omega phase and twin domain can be readily distinguished by the inset $<011>$-zone diffraction patterns. In general, the $\omega$ phase can be identified from the extra diffraction spots excited at various $1 / 3<112>$ and $1 / 3<112>$ positions in the $[011]_{\beta}$-zone pattern as shown in Fig. 2, in which the simulated diffraction pattern for the $\beta$ phase is displayed on the left, and the simulated [11 20 ]-zone pattern of a rumpled $\omega$ phase is displayed on the right. It can be seen readily that the simulated diffraction pattern for rumpled $\omega$ is matching very well with the extra diffraction spots 
appeared at $1 / 3<112>$ and $1 / 3<112>$ positions in the [011 $]_{\beta}$-zone pattern.

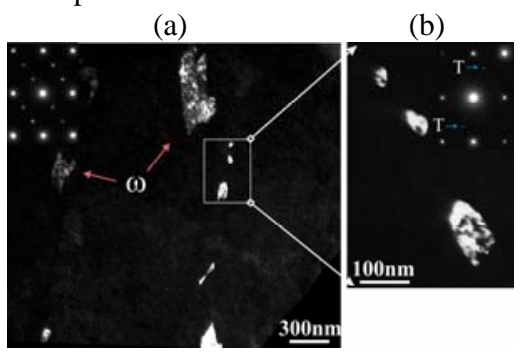

Fig. 1. Dark-field TEM images of (a) an overview and (b) a higher magnification view of the specified region in (a) showing the coexistence of nanoscale twin domains with fine $\omega$ plates within Ta shocked at 50 $\mathrm{GPa}$. Note that diffraction spots corresponding to the twin domains are marked by arrows.

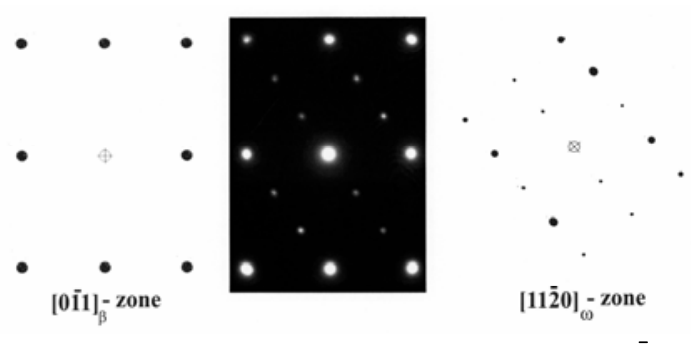

Fig. 2. Selected-area diffraction pattern of the [011]zone generated from a region of $\beta-\omega$ mixture. Simulated diffraction patterns for $\beta$ and rumpled $\omega$ with the corresponding orientations are also displayed.

\section{Unique dislocation structures developed within polycrystalline and single-crystal tantalum}

It has been proposed previously in [8] that the shear operations for the alteration of bcc structure to twin and omega domains can be produced by the glide of partial dislocations of the type $1 / 3<111>, 1 / 6<111>$ and $1 / 12<111>$ dissociated from the $1 / 2<111>$ perfect dislocation. Each $1 / 2<111>$ dislocation can dissociate into three $1 / 6<111>$ partials (each one plane apart) in the $\{211\}$ plane to form a three-layered twin domain. It thus requires the dissociation of two $1 / 2<111>$ dislocations with two-plane apart to form a unit (sixlayer) twin domain. Similarly, the partial dislocations for the $\beta \rightarrow \omega$ transition can be obtained by the dissociation of a $1 / 2[111]$ dislocation into two $1 / 12<111>$ partials and one $1 / 3<111>$ partial (each one plane apart) in the $\{211\}$ plane to form a unit (threelayer) omega domain. These two dissociation reactions, i.e. $\mathbf{b} \rightarrow \mathbf{b}_{1}+\mathbf{b}_{2}+\mathbf{b}_{3}$, are energetically feasible because the free energy is gained in the dissociation process according to Frank's criterion: $b_{1}{ }^{2}+b_{2}{ }^{2}+b_{3}{ }^{2}<b^{2}$. The question is then how these dislocation dissociations contribute to the nucleation of twin domain and omega phase with finite size and thickness. In other words, it is required to precede a dislocation transformation from a one-dimensional line defect to a three-dimensional phase domain, which requires the formation of a high-density dislocation agglomerate in $\{211\}$ with dislocation lines only two-plane apart $(0.27 \mathrm{~nm})$.

A screw dislocation line in reality can contain many atomic jogs [jog height $(\mathrm{d})=0.27 \mathrm{~nm}$ ], which can be either preexisting thermal jogs or generated from the intersection of dislocation lines during shock deformation. It is feasible that under high strain-rate and high-pressure conditions, dislocation loops can multiply and bow out from numerous closely-spaced line segments along a jogged screw dislocation to form dislocation agglomerates as illustrated in Fig. 3(a), in which an elliptical dislocation aggregate resulting from overlapping of closely-spaced loops can be generated when the motion of screw dislocations is largely restricted within polycrystalline tantalum. That is, when a high-density dislocation structure formed within shocked polycrystalline tantalum, the velocity of screw dislocation becomes much slower than that of edge dislocation (i.e., $\mathrm{v}_{\mathrm{s}}<<\mathrm{v}_{\mathrm{e}}$ ). Since edge segments of a loop can only move along the $<111>$-direction, which causes the overlap of numerous closely-spaced loops and results in the formation of dense dislocation clusters that have been observed readily and shown in Fig. 3(b). The dislocation clustering can cause a significant increase of strain energy in local regions of $\beta$ (bcc) matrix, which renders mechanical instability and initiates the nucleation of twin and phase domains through the spontaneous reactions of dislocation dissociation within the dislocation clusters. On the contrary, dislocation clustering is more difficult to take place in shocked single-crystal tantalum because the resulted dislocation density is much lower than that in polycrystalline tantalum shocked at the same conditions. As illustrated in Fig. 3(c), jogged screw dislocation in single-crystal tantalum can move more freely in the direction perpendicular to the $<111>$-direction (i.e., i.e., $\mathrm{v}_{\mathrm{s}}>>\mathrm{v}_{\mathrm{e}}$ ), which results in the formation of dipoles or debris as a result of the non-conservative motion of jogged screw dislocation. A TEM observation of high-density debris generated in single-crystal tantalum shocked at $50 \mathrm{GPa}$ is shown in Fig. 3(d), in which numerous debris appear as white dots in contrast. Neither twin domain nor omega phase was found in the single-crystal tantalum shocked at $50 \mathrm{GPa}$. 
(a)

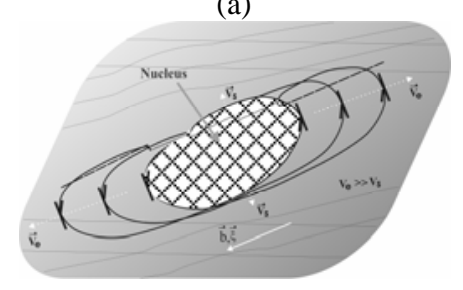

(b)

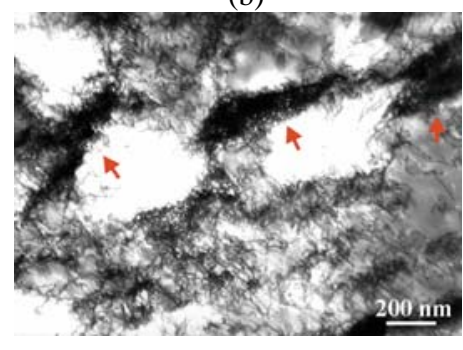

(c)

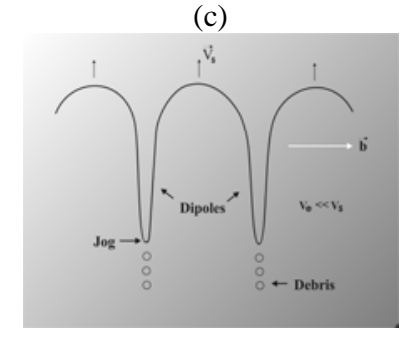

(d)

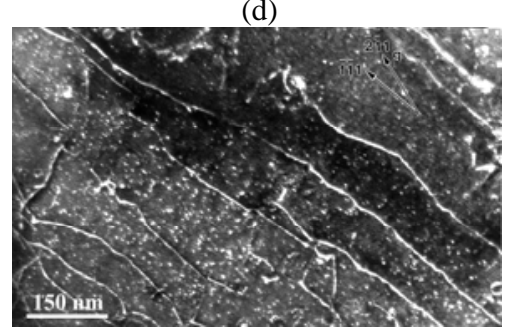

Fig. 3. (a) A schematic illustration of the formation of a dislocation cluster from a jogged screw dislocation within a high-density dislocation structure, where twin or omega phase can be nucleated from the hatched area. (b) TEM image showing a unique dislocation structure containing dense dislocation clusters (marked by arrows) formed in polycrystalline tantalum shocked at $50 \mathrm{GPa}$. (c) A schematic illustration of the debris generated from the non-conservative motion of jogged screw dislocations. (d) TEM image showing a typical dislocation structure with high-density of debris (white dots in contrast) formed in single-crystal tantalum shocked at $50 \mathrm{GPa}$.

\section{CONCLUSION}

Shock-induced martensitic transformation in tantalum has been further investigated. The structure of martensite phase, which formed in polycrystalline tantalum shocked at $50 \mathrm{GPa}$, is identified to be non-ideal hexagonal, namely rumpled $\omega$ phase. It is suggested that shear deformation in the $\{211\}$ planes is the major cause for the formation of shock-induced twin domain and martensite phase. Heterogeneous nucleation mechanisms based upon the dislocation clustering accompanied with the core dissociation of $1 / 2<111>$ into three $1 / 6<111>$ twinning dislocations, and $1 / 12<111>, 1 / 3<111>$ and $1 / 12<111>$ transformation dislocations are further verified to be vital micromechanisms for the shockinduced twinning and phase transformation in tantalum.

\section{ACKNOLEDGEMENTS}

This work was performed under the auspices of the U. S. Department of Energy by the University of California, Lawrence Livermore National Laboratory under Contract No. W-7405-Eng-48. The author would like to thank Dr. David Lassila for providing shock-recovered tantalum samples for this investigation.

\section{REFERENCES}

1. Huang, J. C., Gray III, G. T., Acta Metall. 37, 3335 (1989).

2. Muff, L. E., Niou, C.-S., Pappu, S., Rivas, J. M., Quinones, S. A., Phys. Stat. Solids A. 149, 253 (1995). 3. Murr, L. E., Meyers, M. A., Niou, C.-S., Chen, Y. J., Pappu, S., Kennedy, C., Acta Mater. 45, 157 (1997).

4. Nesterenko, V. F., Meyers, M. A., LaSalvia, J. C., Bondar, M. P., Chen, Y. J., Lukyanov, Y. L., Mater. Sci. Eng. A229, 23 (1997).

5. Briant, C. L., Batcheler, R. H., Lassila, D. H., Gourdin, W., in Tantalum, edited by Chen et al., p. 191, TMS, Warrendale, PA (1996).

6. Lassila, D. H., Gray III, G. T., J. Phys. IV, Colloque C3, 1, C3-19 (1991).

7. L.M. Hsiung and D.H. Lassila, Scripta Mater. 39, 603 (1998).

8. Hsiung, L. M., Lassila, D. H., Acta Mater. 48, 4851 (2000).

9. Young, D. A., Phase Diagrams of the Elements, University of California Press, Berkeley: California, 1991.

10. Tonkov, E. Yu, Ponyatovsky, E. G., Phase Transformations of Elements under High Pressure, CRC Press, 2005.

11. S. K. Zikka, Y. K. Vohra, and R. Chidambaram, Progress in Materials Science 27, 245 (1982). 